\title{
Nuevos registros de taxones de interés corológico para la flora vascular de la provincia de Córdoba (Andalucía occidental, España)
}

\author{
Rafael Tamajón Gómez, Blanca Rodríguez Linares y Sara Parras León \\ Departamento de Botánica, Ecología y Fisiología Vegetal, Facultad de Ciencias, Universidad de Córdoba. Edificio José \\ Celestino Mutis, Campus de Rabanales, Universidad de Córdoba, 14071 (Córdoba).
}

\section{Correspondencia}

Rafael Tamajón Gómez

e-mail: pseudicius@hotmail.com

Recibido: 22 mayo 2021

Aceptado: 1 septiembre 2021

Publicado on-line: 4 octubre 2021

Editado por: Marta Recio Criado

\begin{abstract}
Resumen
Se aportan nuevos registros de varios taxones de flora vascular, tanto autóctonos como alóctonos naturalizados, de interés corológico, herborizados en la provincia de Córdoba (España): Vachellia farnesiana (L.) Wight \& Arn. (novedad para Andalucía occidental), Astragalus boeticus L. (novedad para la Vega y para la provincia de Córdoba), Cullen americanum (L.) Rybd. (novedad para la Vega y Sierra Norte), Solanum elaeagnifolium Cav. (novedad para la provincia de Córdoba) y Pulicaria dysenterica (L.) Bernh. (con escasos registros previos provinciales en Sierra Norte, Campiña Alta y Subbética, se amplía su área de distribución conocida dentro de la Vega, ya que la localidad previa más cercana se sitúa a unos $100 \mathrm{~km}$ de distancia, en la provincia de Sevilla). Todos los pliegos testigo están depositados en el Herbario del Real Jardín Botánico de Córdoba (COA).
\end{abstract}

\begin{abstract}
New records of taxa with chorological interest for the flora of Cordoba province (Western Andalusia, Spain).

New records of some taxa of vascular flora collected in Cordoba province (Spain), both native and naturalized, with chorological interest, are provided here. They represent novelties for both Western Andalusia (Vachellia farnesiana (L.) Wight \& Arn.), and several natural regions (Astragalus boeticus L., new for La Vega and for Cordoba province, and Cullen americanum (L.) Rybd., new for La Vega and Sierra Norte) or just for Cordoba province (Solanum elaeagnifolium Cav.); in the case of Pulicaria dysenterica (L.) Bernh, with few previous province records, from Sierra Norte, Campiña Alta and Subbética, its known range has been enlarged (the nearest known population within La Vega is $100 \mathrm{~km}$ far from the new one, in the province of Seville). All the sheets are deposited in the Herbarium of the Royal Botanical Garden of Cordoba (COA).
\end{abstract}

\section{Palabras clave}

España, Andalucía occidental, Córdoba, flora vascular, flora exótica, corología.

Key words

Spain, Western Andalusia, Cordoba, vascular flora, alien flora, chorology.
El objeto de la presente nota es contribuir al conocimiento de la flora vascular de la provincia de Córdoba (España). Para ello se aportan nuevos registros de varios taxones de interés corológico, tanto autóctonos como alóctonos naturalizados, herborizados recientemente en dicho territorio. Para la denominación de las comarcas naturales citadas en el texto se ha seguido la propuesta de sectorización empleada en la Flora Vascular de Andalucía Occidental (Valdés et al., 1987).

Astragalus boeticus L., Sp. PI. 738 (1753)

CÓRDOBA. Córdoba capital, en un descampado, 30SUG49, coordenadas geográficas: $37^{\circ} 52^{\prime} 36,1^{\prime \prime}$ $\mathrm{N}, 4^{\circ} 46^{\prime}$ 19,2" W, 97 m.s.n.m., 11-III-2020, $R$. Tamajón (COA 60382) (Figura 1).
El café de los pobres o garbancillo, como se conoce vulgarmente a $A$. boeticus, es una fabácea que se distribuye por las regiones Mediterránea, Irano-Turaniana y Macaronésica; en el caso concreto de la Península Ibérica se localiza en la franja costera meridional, desde Lisboa hasta Castellón, pero su área también incluye las Islas Baleares (Podlech, 1999). En el siglo XIX se cultivó en varios países de Europa, en especial en Suecia, como sucedáneo del café (Prohens et al., 2014); en la Península Ibérica contamos con una referencia antigua relativa a su cultivo en el NE de España y a su naturalización en las ramblas del Río Gállego, en Zaragoza, y en los campos de Barcelona (Willkomm \& Lange, 1877).

En Andalucía occidental no hay constancia de su 
cultivo y se conoce su presencia previa en el Litoral de Huelva (Sánchez Gullón, 1999; López-Albacete et al., 2007; Valdés et al., 2007) y de Cádiz (Colmeiro, 1886; Domínguez, 1987), así como en la provincia de Sevilla, donde aparece citada sin indicación de localidad geográfica precisa (Colmeiro, 1886), y también en Los Alcores (Medina, 1891; Galiano \& Valdés, 1976) y en la Campiña Baja Sevillana (César Cruz, 2011). Según este último autor, las referencias más antiguas acerca de su existencia en la Campiña Baja sevillana (Domínguez, 1987) deberían atribuirse en realidad a la comarca de Los Alcores (César Cruz, 2011). El presente hallazgo es una novedad para la provincia de Córdoba y al mismo tiempo constituye el primer registro para la comarca de La Vega.

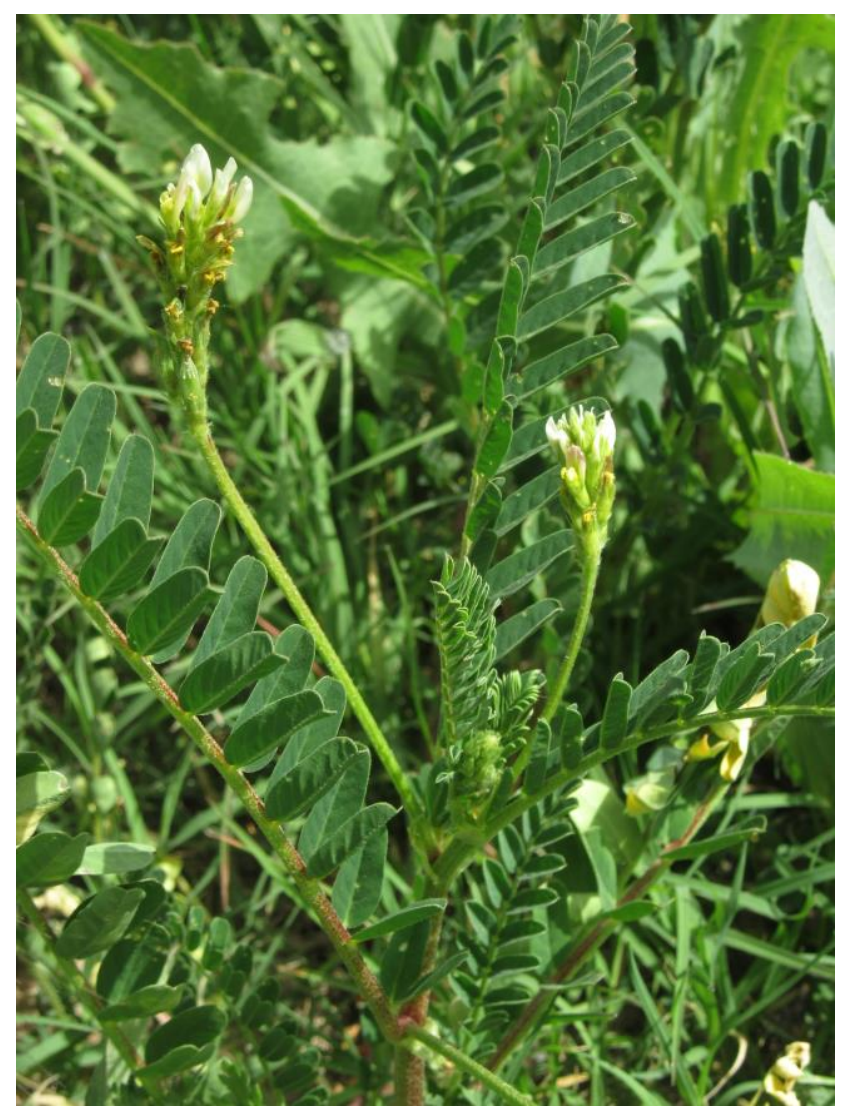

Figura 1. Astragalus boeticus, ejemplar en floración (31-V2021).

Figure 1. Astragalus boeticus, specimen in flowering (31V-2021).

En el censo llevado a cabo en el año 2020 se contabilizaron 158 ejemplares, tanto aislados como creciendo en rodales (de hasta un máximo de 65 individuos), que se concentran en el borde meridional del descampado. Un censo reciente (20IV-2021) ha arrojado una cifra de tan solo 31 ejemplares. La vegetación de este enclave es un herbazal subnitrófilo bastante heterogéneo en cuanto a composición florística y altura. En la zona del baldío donde se concentra la población de $A$. boeticus existe predominio de las gramíneas, entre las que destacan Avena sterilis L. y varias especies del género Bromus L., pero también es muy abundante la asterácea Glebionis coronaria (L.) Spach. y los jaramagos del género Diplotaxis DC., entre otras especies ruderales.

Cullen americanum (L.) Rybd. in Britton, N. Amer. FI. 24: 3 (1919)

CÓRDOBA. Córdoba capital, C/ Vereda del Pretorio, 30SUG49, coordenadas geográficas: $37^{\circ} 55^{\prime} 28,6^{\prime \prime}$ $\mathrm{N}, 4^{\circ} 48^{\prime}$ 08,3" O, 266 m.s.n.m., 9-VIII-2019, $R$. Tamajón (COA 60712); Córdoba capital, C/ Cordel de Écija, 30SUG49, coordenadas geográficas: $37^{\circ}$ 51' 49,3" N, 4 47' 03.5" O, 94 m.s.n.m., 30-IV-2021, R. Tamajón (COA 60718).

La higueruela ( $C$. americanum) es una leguminosa de carácter arvense que se distribuye por Madeira y el oeste de la Región Mediterránea, incluyendo la Península Ibérica, Marruecos, Argelia, Túnez y Malta (Talavera, 1999). Según Talavera (1999), es nativa del sur de España -Extremadura y Andalucía- pero también aparece en otros territorios, como adventicia (Tarragona) o como naturalizada (Madrid, Portugal).

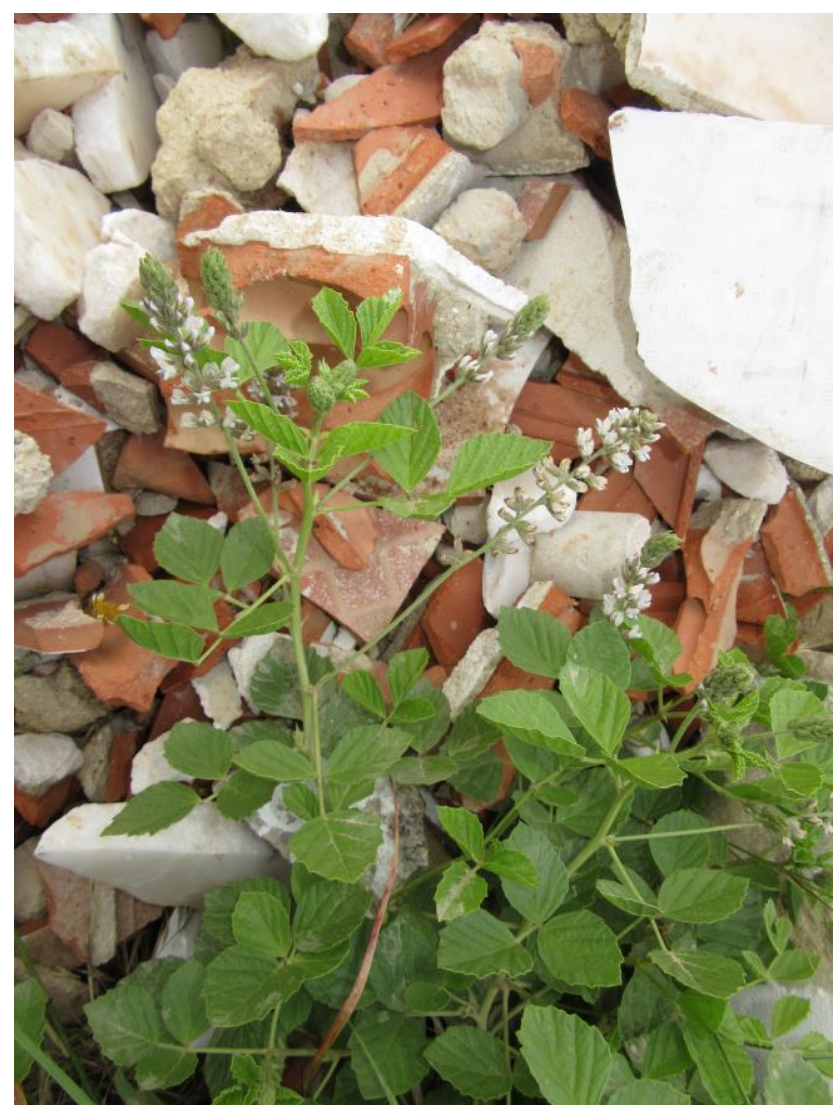

Figura 2. Cullen americanum, ejemplar en floración, creciendo entre escombros (29-IV-2021).

Figure 2. Cullen americanum, specimen in flowering, growing among rubble (29-IV-2021).

Inicialmente citada en Andalucía occidental tan sólo de las provincias de Cádiz y Sevilla (Domínguez, 1987), hasta el momento existen registros publicados para Cádiz (Pérez, 1892; Willkomm, 1893; Rigueiro, 1978; Monge \& Carrasco, 1990), Sevilla (Galiano \& Valdés, 1976; Ruíz de Clavijo et al., 1984; Monge \& Carrasco, 1990), 
Huelva (Sánchez Gullón et al., 1999; Talavera, 1999) y Córdoba. En esta última provincia se había citado previamente en tres ocasiones, pero siempre de comarcas situadas al sur del Guadalquivir: Subbética (Triano, 2010), Campiña Baja (López Tirado et al., 2013) y Campiña Alta (Muñoz Álvarez et al., 2016).

Los nuevos registros que aquí se presentan constituyen una novedad tanto para la comarca de La Vega (el de menor altitud, próximo al Guadalquivir) como para la de Sierra Norte (el de mayor altitud, ya en el inicio de la falda de la Sierra de Córdoba, en la zona de transición con la comarca de la Vega). En ambas localidades las plantas crecen sobre sustratos básicos (suelos arcillosos sobre calizas cámbricas y sustratos aluviales, respectivamente); en el caso concreto del ejemplar del Cordel de Écija, en la zona se acumulan frecuentemente escombros (Figura 2), y entre las plantas acompañantes -que son típicas de comunidades herbáceas ruderales y viariasdestacan por su abundancia Piptatherum miliaceum (L.) Coss. e Hirschfeldia incana (L.) Lagr.-Foss., y en menor medida, Glebionis coronaria (L.) Cass. ex Spach, Sinapis alba subsp. mairei (H. Lindb.) Maire, Avena barbata Pott ex Link., A. sterilis L., Vulpia geniculata (L.) Link., Hordeum murinum subsp. leporinum (Link.) Arcang., Malva multiflora (Cav.) Soldano, Banfi \& Galasso, Daucus carota L., Andryala integrifolia L., Amaranthus muricatus (Gillies ex Moq.) Hieron y Erigeron bonariensis L.

Pulicaria dysenterica (L.) Bernh. Syst. Verz. Erfurt 153 (1800)

CÓRDOBA. Córdoba capital, Monumento Natural "Sotos de la Albolafia", 30SUG49, coordenadas geográficas: $37^{\circ} 52^{\prime} 29,3 " \mathrm{~N} \quad 4^{\circ} \quad 46^{\prime} 51,6 " \mathrm{O}$, 88 m.s.n.m., 7-VIII-2019, R. Tamajón (COA 60711).

Especie latecircunmediterránea (Pujadas Salvá, 1986), distribuida por la mayor parte de Europa excepto el extremo septentrional-, así como por el norte de África y suroeste de Asia (Devesa, 1987). En la Península lbérica esta asterácea se distribuye fundamentalmente en la mitad oriental, y en concreto en el cuadrante noreste, considerando la concentración de citas publicadas (Anthos, 2021).

Si bien en la Flora Vascular de Andalucía Occidental se consideraba común en ambientes húmedos de todo el territorio (Devesa, 1987), los registros publicados son muy escasos (Anthos, 2021). En el caso de la provincia de Córdoba las únicas referencias previas han sido aportadas por Pujadas Salvá (1986), que cita la especie de las comarcas Sierra Norte, Campiña Alta y Subbética. La nueva población localizada constituye el segundo registro andaluz para la comarca de la Vega, y se sitúa a más de $100 \mathrm{Km}$ respecto del primero, en la provincia de Sevilla (Barras de Aragón, 1899). La población localizada crece en herbazales higronitrófilos dominados por el mastranzo (Mentha suaveolens Ehrh.), muy cerca del cauce del río Guadalquivir.
Solanum elaeagnifolium Cav., Icon. 3:22, tab. 243 (1795)

CÓRDOBA. Subestación de Los Cansinos, en los restos del antiguo Cortijo de la Rinconada, 30SUG59, coordenadas geográficas: $37^{\circ} 55^{\prime} 34,0^{\prime \prime}$ $\mathrm{N}, 4^{\circ} 35^{\prime} 44,2^{\prime \prime}$ O, 118 m.s.n.m., 19-VII-2020, R. Tamajón (COA 60713) (Figura 3).

Especie alóctona, de carácter ruderal y viaria, originaria de América, que aparece como introducida y naturalizada en el este de Estados Unidos, así como en varios países europeos -entre ellos España-, Egipto, Sinaí, India y África austral (Sobrino \& Sanz Elorza, 2012). En España esta solanácea se encuentra naturalizada principalmente en áreas litorales de Cataluña y Levante, pero también se conoce de varias localidades del suroeste (Extremadura y Andalucía) y del interior Madrid y Aragón- (Sobrino \& Sanz Elorza, 2012; Anthos, 2021). En Andalucía todas las citas previas proceden exclusivamente de dos provincias occidentales, Sevilla y Huelva. Los registros de Sevilla se corresponden con localidades de las comarcas de la Vega y de Los Alcores (Aparicio, 2003; Jiménez Mejías et al., 2010), y el único de Huelva procede de Lucena del Puerto, en la comarca del Litoral onubense (Sánchez Gullón et al., 2006). La presente cita constituye una novedad para la flora de la provincia de Córdoba y además supone la ampliación del área conocida dentro de la comarca de La Vega, ya que la nueva población localizada se sitúa más de $130 \mathrm{~km}$ al este de las localidades sevillanas previamente registradas.

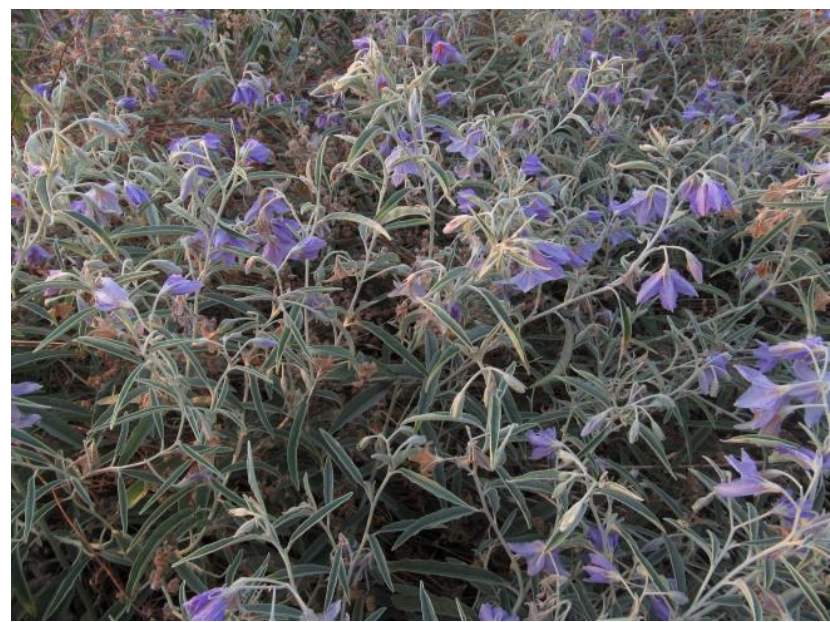

Figura 3. Solanum elaeagnifolium, rodal en floración (19VII-2021).

Figure 3. Solanum elaeagnifolium, flowering stand (19-VII2021).

La población naturalizada hallada es muy densa y ocupa una superficie total estimada de unos 200$300 \mathrm{~m}^{2}$. La altura media de las plantas está en torno a 0,5-0,75 m. Esta especie crece en un terreno llano, colonizado por un herbazal nitrófilo muy heterogéneo, que se desarrolla sobre el antiguo asentamiento de una edificación rural desaparecida (el Cortijo de la Rinconada), de la cuál tan sólo quedan algunos restos de soleras y un pequeño 
muro de piedra. Esto podría explicar su posible origen, un escape a partir de una población cultivada como ornamental. Entre las especies acompañantes destacan por su abundancia Piptatherum miliaceum (L.) Coss., Avena barbata Pott ex Link y Lactuca serriola L., pero también pueden citarse las siguientes: Hirschfeldia incana (L.) Lagr.-Foss., Bromus madritensis L., Carduus tenuiflorus Curtis, Andryala integrifolia L., Sinapis alba subsp. mairei (H. Lindb.) Maire, Vulpia geniculata (L.) Link, Silybum marianum (L.) Gaertn., Carthamus lanatus L. y Marrubium vulgare L. Este herbazal está rodeado de un pequeño olivar, pero también hay pies dispersos de Morus alba L. y Gleditsia triacanthos L. En el entorno, de carácter agrícola, encontramos extensas áreas de vega dedicadas a cultivos de regadío. Teniendo en cuenta los ambientes colonizados -por el momento exclusivamente ruderales- en la provincia de Córdoba se comporta como un metáfito epecófito, según la clasificación de Kornas (1990), xenotipo que ya le había sido asignado a nivel genérico en España por Sanz Elorza et al. (2004).

Vachellia farnesiana (L.) Wight \& Arn. Prodr. fl. Ind. orient. 272. 1834

[Acacia farnesiana (L.) Willd]

CÓRDOBA. Córdoba capital, junto al Canal del Guadalmellato (Figura 4), 30SUG49, coordenadas geográficas: $37^{\circ} 54^{\prime} 13,0^{\prime \prime} \mathrm{N}, 4^{\circ} 46^{\prime} 24,6^{\prime \prime} \mathrm{O}, 135$ m.s.n.m, 2-IX-2019, R. Tamajón (COA 60714); CÓRDOBA. Alcolea, junto a las vías del ferrocarril, 30SUG59, coordenadas geográficas: $37^{\circ} 55^{\prime} 58,8^{\prime \prime}$ $\mathrm{N}, 4^{\circ} 40^{\prime}$ 33,2" O, 112 m.s.n.m., 7-IX-2020, $R$. Tamajón (COA 60715); ibidem, 8-IV-2021, $R$. Tamajón (COA 60717).

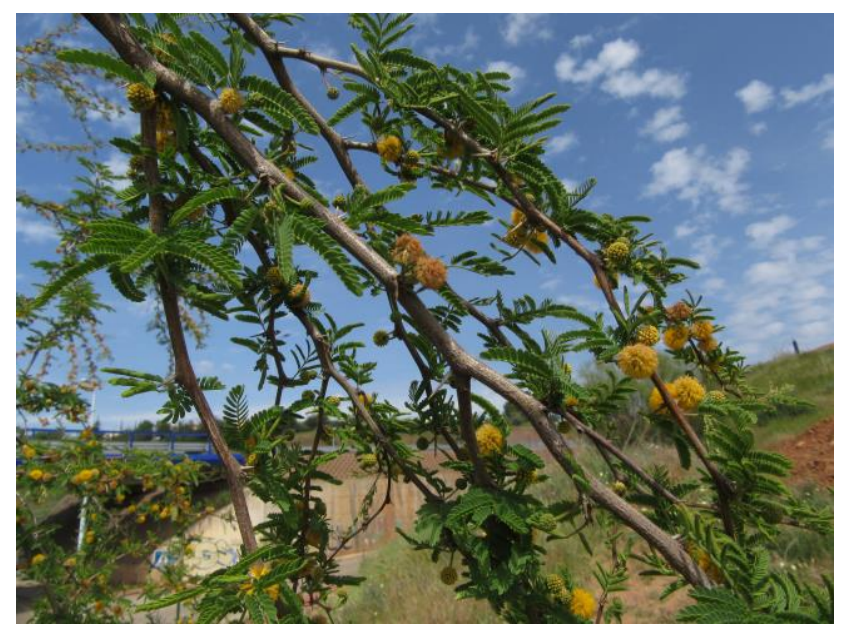

Figura 4. Vachellia farnesiana, individuo con ramas florecidas, Córdoba capital (13-IV-2021).

Figure 4. Vachellia farnesiana, specimen with flowering branches, Cordoba city (12-IV-2021).

Esta fabácea arbustiva espinosa y caducifolia es probablemente originaria de América Tropical y aparece introducida en otras regiones tropicales y subtropicales; en la actualidad presenta una distribución pantropical, con representación en algunas áreas templadas (Paiva, 1999). Hasta el momento, en la Península lbérica se había citado como naturalizada en áreas del sureste y del este (Paiva, 1999; Anthos, 2021), en concreto en las provincias de Almería (Ruíz de la Torre, 1979), Murcia (Martínez et al., 2008), Alicante (Serra, 2007), Valencia (Borja, 1951; Herrero-Borgoñón, 2009), Castellón (Roselló \& Peris, 1983; Herrero-Borgoñón, 2009; Royo, 2006) y Tarragona (Royo, 2006). Por consiguiente, las poblaciones localizadas suponen una novedad corológica no sólo para la flora de la provincia de Córdoba y la comarca de la Vega, sino también para Andalucía occidental.

Se han localizado dos poblaciones que distan unos $10 \mathrm{~km}$ entre sí, ambas en ambientes antropizados. La más importante es la de la periferia del núcleo urbano de Alcolea, que consta de dos subpoblaciones. El núcleo principal cuenta con ocho individuos, dos de ellos adultos -con alturas diferentes, uno de $7 \mathrm{~m}$ y otro de $3 \mathrm{~m}$ - y el resto son plantones jóvenes procedentes de semilla; a menos de $100 \mathrm{~m}$ se ha localizado creciendo aislado otro ejemplar adulto de porte mediano. La otra población, en Córdoba capital, cuenta en la actualidad con un único ejemplar adulto, de unos $5 \mathrm{~m}$ de altura (estaba acompañado por otro ejemplar adulto, de unos 2,75 $3 \mathrm{~m}$, pero ha sido eliminado a raíz de unas obras recientes).

Aunque a nivel global en España Sanz Elorza et al. (2004) asignan a esta especie la categoría de metáfito hemiagriófito -según la terminología y clasificación de los xenófitos propuesta por Kornas (1990)-, en el caso concreto de Córdoba se está comportando por el momento como metáfito epecófito, teniendo en cuenta la tipología de los ambientes ocupados, que no son seminaturales sino de carácter ruderal y viario (respectivamente un bosquete de Ailanthus altissima (Miller) Swinger, en los bordes de una línea de ferrocarril, y un herbazal nitrófilo, en el talud de una carretera y en el borde de un canal de riego), en las afueras de varios núcleos de población.

\section{AGRADECIMIENTOS}

A nuestro compañero Diego Peinazo, de la Sociedad Cordobesa de Historia Natural (SCHN), que amablemente nos proporcionó la ubicación geográfica de la población de Solanum elaeagnifolium que había encontrado por casualidad. A Emilio Laguna, Antonio Pujadas y Javier López Tirado por revisar el borrador del manuscrito y por sus comentarios y sugerencias sobre el mismo. A Paco Muñoz, por facilitarnos la consulta de cartográfica histórica así como de las series de fotografías aéreas disponibles para documentar la existencia, localización y evolución del antiguo Cortijo de la Rinconada.

\section{BIBLIOGRAFÍA}

Anthos. 2021. Sistema de Información de las plantas de España. Real Jardín Botánico, CSIC - 
Fundación Biodiversidad. Recurso electrónico disponible en línea en: http://www.anthos.es. Con acceso el 11-IV-2021.

Aparicio, A. (2003). Dos taxones naturalizados en la Península Ibérica presentes en Andalucía Occidental. Acta Botanica Malacitana, 28. 253. https://doi.org/10.24310/abm.v28i0.7294

Barras de Aragón, F. de las (1899). Datos para la flórula sevillana (Continuación). Actas Soc. Esp. Hist. Nat., 28, 255-261.

Borja Carbonell, J. (1951). Estudio fitográfico de la Sierra de Corbera (Valencia). Anales Jard. Bot. Madrid, 9, 361-483.

https://dialnet.unirioja.es/descarga/articulo/29701 48.pdf

César Cruz, M. (2011). Nueva cita corológica para Astragalus boeticus. Lagascalia, 31, 239-240. http://institucional.us.es/revistas/lagascalia/31/not as.pdf

Colmeiro, M. (1886). Enum. Pl. Peníns. HispanoLusit. Tomo II (Calcifloras: Sección 19a). Imprenta de la viuda e hija de Fuentenebro, Bordadores, 10. Madrid, $642 \mathrm{pp}$.

Devesa, J. (1987). Pulicaria, in Valdés, B., Talavera, S. \& Galiano, E. (Eds.), Flora Vascular de Andalucía Occidental, 3 (pp.18-20). Ketres. Barcelona.

Domínguez, E. (1987). Astragalus, In Valdés B., Talavera, S. \& Galiano, E. (Eds.), Flora Vascular de Andalucía Occidental, 2 (pp. 182-188). Ketres. Barcelona.

Galiano, E. \& Valdés, B. (1976). Catálogo de plantas vasculares de la provincia de Sevilla. VIII Rosales (Papilionaceae). Lagascalia, 6(1), 39-89.

Herrero-Borgoñón, J. (2009). Notas sobre flora alóctona valenciana. Flora Montiberica, 49: 19-25. https://dialnet.unirioja.es/descarga/articulo/30445 66.pdf

Jiménez-Mejías, P., Escudero, M. \& Martín Bravo, S. (2010). Notas sobre neófitos en el curso urbano del río Guadalquivir en Sevilla. Lagascalia, 30, 472-481. https://hdl.handle.net/11441/87444

Kornas, J. (1990). Plant invasions in Central Europe: historical and ecological aspects. In: Di Castri, F., A.J. Hansen \& M. Debussche (eds.) Biological Invasions in Europe and in the Mediterranean Basin, pp. 19-36. Kluwer Academic Publishers. The Netherlands.

López-Albacete, I., de las Heras, M.A., SánchezGullón, E., Weickert, P., Hidalgo, P.J. \& Muñoz, A.F. (2007). Nuevas localidades de especies interesantes en Doñana y la costa de Huelva (SW España). Acta Botanica Malacitana, 32, 258261. doi: https://doi.org/10.24310/abm.v32i0.7050

López Tirado, J., Muñoz Álvarez, J. M. \& Hidalgo, P. J. (2013). Aportaciones a la flora vascular de la provincia de Córdoba I (Andalucía, España). Lagascalia, 33, 314-325.

http://institucional.us.es/revistas/lagascalia/33/not as.pdf

Martínez Sánchez, M., Fernández, S. \& Carrión, J. (2008). Palinología y escenario forense. Un caso de estudio del sureste de España. Anales de Biología, 30, 43-54.

https://www.um.es/analesdebiologia/numeros/30/ PDF/30_05.pdf

Medina, $\bar{M}$. (1891). Excursions botaniques en Espagne. Actas Real Soc. Esp. Hist. Nat., 20, 122-125.

https://doi.org/10.1080/00378941.1882.10828082

Monge, C. \& Carrasco, A. (1990). Cartografía Corológica Ibérica. Aportación 10, Botanica Complutensis, 16, 131.

https://revistas.ucm.es/index.php/BOCM/article/d ownload/BOCM9090110129A/6548

Muñoz, J.M., Vega, R. F., Delgado, J. M., Díaz, M. L. \& Alcántara, J. (2016). Contribución a la flora vascular de los humedales del sur de Córdoba (Andalucía, España). Flora Montiberica, 65, 8-16. http://www.floramontiberica.org/FM/Flora_Montib erica65.pdf\#page $=11$

Paiva, J. (1999). Acacia Mill. In Castroviejo S., Aedo, C., Laínz, M., Muñoz Garmendia, F., Nieto Feliner, G., Paiva, J. \& Benedí, C. (Eds.), Flora iberica, 7(2) (pp.11-25). Real Jardín Botánico, CSIC. Madrid.

Pérez Lara, J.M. (1892). Florula gaditana. Pars quarta. (Continuación). Anales Soc. Esp. Hist. Nat., 21(2), 191-280.

Podlech, D. (1999). Astragalus L. In Castroviejo S., Aedo, C., Laínz, M., Muñoz Garmendia, F., Nieto Feliner, G., Paiva, J. \& Benedí, C. (Eds.), Flora Iberica, 7(1): (pp. 279-338). Real Jardín Botánico, CSIC. Madrid.

Prohens, J., Andújar, I., Vilanova, S., Plazas, M., Gramazio, P., Prohens. R., Herraiz, F. J. \& De Ron, A. M. (2014). Swedish coffee (Astragalus baeticus $\mathrm{L}$.), a neglected coffee substitute with a past and a potential future. Genet. Resour. Crop Evol. 61, 287-297. https://doi.org/10.1007/s10722-013-0059-0

Pujadas Salvá, A. (1986). Flora arvense y ruderal de la Provincia de Córdoba. Tesis Doctoral. Universidad de Córdoba.

Rigueiro Rodríguez. A (1978). Catálogo florístico de la Sierra de Grazalema. Trab. Catedra Bot. Esc. Tecn. Super. Ing. Montes 4 [I-XVIII], 1-272.

Roselló, R. \& Peris, J.B. (1983). Algunos neófitos de la provincia de Castellón. Fontqueria, 28, 53-56.

Royo Pla, F. (2006). Flora i vegetació de les planes i serres litorals compreses entre el riu Ebro i la serra d'Irta. Tesis Doctoral. Universitat de Barcelona, Facultat de Biologia.

Ruiz de Clavijo, E., Cabezudo, B. \& Domínguez, E. (1984). Contribución al estudio florístico de las serranías subbéticas de la provincia de Sevilla. Acta Botanica Malacitana, 9, 169-232.

https://doi.org/10.24310/Actabotanicaabmabm.v9i .9641

Ruiz de la Torre, J. (1979). Notas de flora hispanica. Trab. Cátedra Bot. Esc. Tecn. Super. Ing. Montes 5, 1-39.

Sánchez Gullón, E. (1999). Novedades corológicas para la provincia de Huelva. Acta Botanica 
Malacitana, 24, 242-247. https://doi.org/10.24310/abm.v24i0.8536

Sanz Elorza, M., Dana Sánchez, E. D. \& Sobrino Vesperinas, E. (Eds.) (2004). Atlas de las Plantas Alóctonas Invasoras en España. Dirección General para la Biodiversidad, Ministerio de Medio Ambiente, Madrid. 384 pp.

Serra Laliga, L. (2007). Estudio crítico de la flora vascular de la provincia de Alicante: aspectos nomenclaturales, biogeográficos y de conservación. Ruizia, 19, 1-1414.

Sobrino Vesperinas, E. \& Sanz Elorza, M. (2012). Solanum L. in Talavera, S., Andrés, C., Arista, M., Fernández Piedra M.P., Gallego, M.J., Ortiz, P.L., Romero Zarco, C., Salgueiro, F.J., Silvestre, S. \& Quintanar, A. (Eds.), Flora iberica, 11 (pp. 166195). CSIC. Madrid.

Talavera, S. (1999). Cullen Medik. in Talavera, S.,
Aedo Pérez, C., Castroviejo Bolibar, S., Romero Zarco, C., Sáez i Goñalons, L, Salgueiro González, F.J. \& Velayos Rodríguez, M. (Eds.), Flora iberica, 7(1) (pp.357-360). CSIC. Madrid.

Triano, E. (2010). Nueva Flora del Subbético cordobés. Formato digital (DVD).

Valdés, B., Girón, V., Sánchez Gullón, E. \& Carmona, I. (2007). Catálogo florístico del espacio natural de Doñana (SO de España). Plantas Vasculares. Lagascalia, 27, 73-362. https://idus.us.es/handle/11441/54114

Valdés, B., Talavera, S. \& Galiano, E. (Eds.) (1987). Flora Vascular de Andalucía Occidental, 1. Ketres. Barcelona. 486 pp.

Willkomm, M. \& Lange, J. (1877). Prodr. Fl. Hispan. Vol. 3(3). Stuttgartiae. E. Schweizerbart. 1144 pp.

Willkomm, M. (1893). Supplementum Prodromi Florae Hispanicae. 